\title{
RELCASI
}

\section{Utilización de la Metodología Mixta de Investigación en Sistemas de Información: El caso del estudio de la Cultura Ocupacional de Sistemas}

Indira R. Guzmán

TUI University \& Universidad Católica Boliviana, iguzman@tuiu.edu

\section{Recommended Citation}

Guzmán, Indira R. (2008) "Utilización de la Metodología Mixta de Investigación en Sistemas de Información: El caso del estudio de la Cultura Ocupacional de Sistemas," RELCASI: Vol. 1 : Iss. 1 , Paper 1. DOI: $10.17705 / 1$ relc.00002

Available at: https://aisel.aisnet.org/relcasi/vol1/iss1/2

This material is brought to you by the AIS Journals at AIS Electronic Library (AISeL). It has been accepted for inclusion in RELCASI by an authorized administrator of AIS Electronic Library (AISeL). For more information, please contact elibrary@aisnet.org. 


\section{$\begin{array}{lllllllll}\text { R I L C A S I } & 01 & 01 & 2008\end{array}$}

\section{REVISTA LAINOAMERICANA}

Y DEL CARIBE DE LA

ASOCIACIÓN DE SISIEMAS

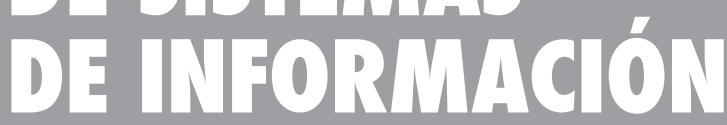

11 - Utilización de la Metodología

Mixta de Investigación en SI:

El caso del estudio de la Cultura

Ocupacional de Sistemas Indira R. Guzmán

31 - Estudio Exploratorio de los Departamentos de Tecnologías de Información Juan Manuel Gómez Reynoso, Carlos Alejandro Torres Quiñones y Javier Santiago Cortes López

53 - Factores Decisivos para el Éxito de la Capacitación Corporativa con Base en la Web en Brasil: Una Investigación Exploratoria Multicaso Luiz Antonio Joia y Mário Figueiredo Costa

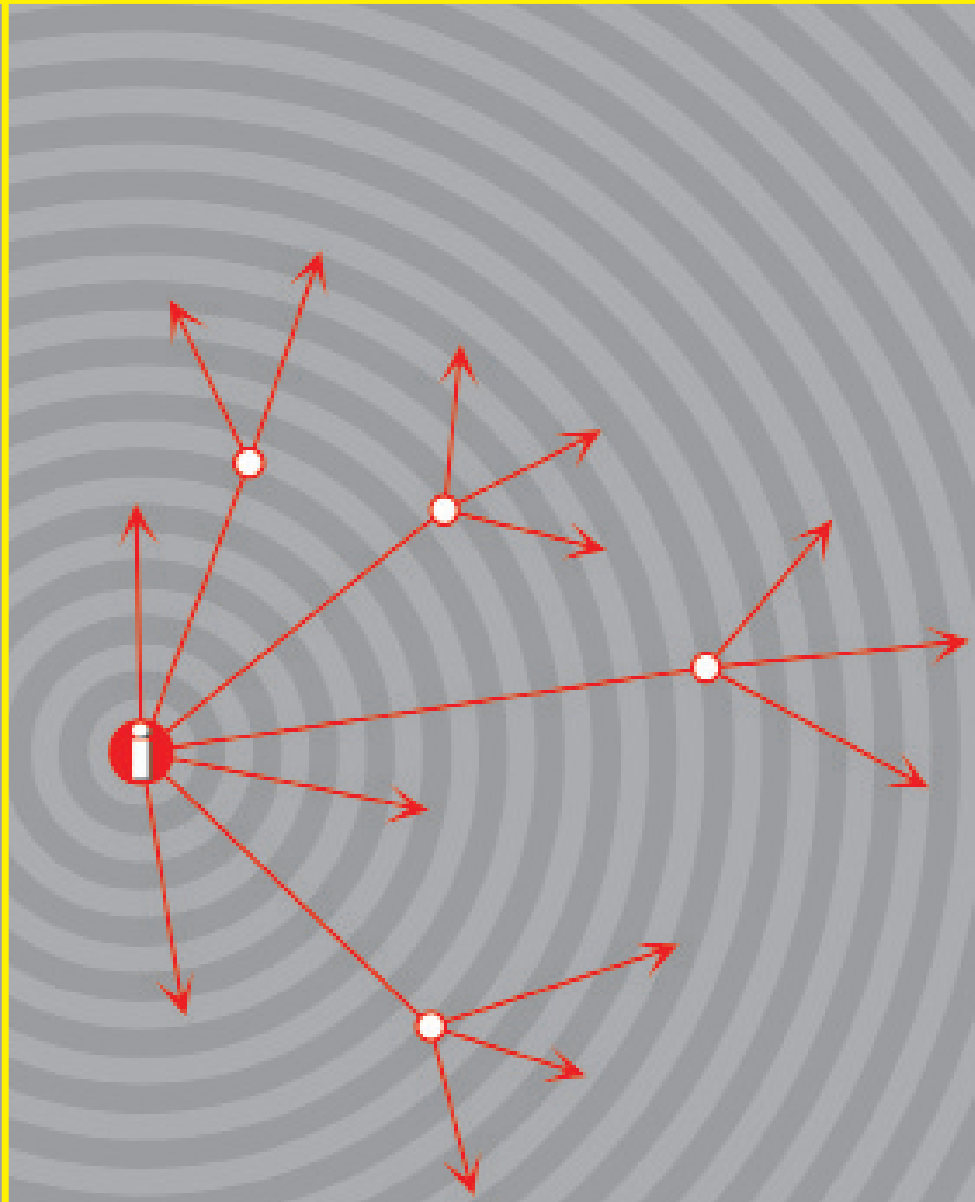




\section{$\begin{array}{lllllllllll}\mathbf{R} & \mathbf{E} & \mathbf{L} & \mathbf{C} & \mathbf{A} & \mathbf{S} & \mathbf{I} & & 01 & 01 & 2008\end{array}$}

\section{REVISTA LAIINOAMERICANA}

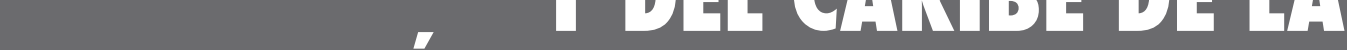
ASOCIACIÓN DE SISTIMAS DE INFORMAción

Editor:

Carlos Ferran 
Carlos Fe

Editor:

Penn State Great Valley Pennsylvania State University 30 E Swedesford Road

Malvern, PA 19355

U.S.A.

cferran@psu.edu

Comité Editorial:

Carlos Dorantes

Tecnológico de Monterrey, México

cdorante@itesm.mx

Carlos Ferran

Pennsylvania State University,

USA

cferran@psu.edu

Martha Garcia-Murillo

Syracuse University, USA

mgarciam@syr.edu

David Montesinos

INCAE, Costa Rica

Montesin@mail.incae.ac.cr

Carlos J. Navarrete California State Polytechnic University, USA

cjnavarrete@csupomona.edu

James B. Pick

University of Redlands, USA

James_Pick@redlands.edu

Guillermo Rodríguez-Abitia

Tecnológico de Monterrey,

Campus Estado de México

grdrz@itesm.mx

Martin Santana

ESAN, Peru

MSantana@esan.edu.pe

Volumen 1 Número 1, 2008

Portada: Maria Elena Repiso

(c) 2008 RELCASI

ISSN 1937-8823

(en línea) ISSN 1937-8831

www.relcasi.org

\section{Revista Latinoamericana} y del Caribe de la Asociación de Sistemas de Información RELCASI

\section{TABLA DE CONTENIDO}

Volumen 1 Número 1, 2008

INTRODUCCIÓN EDITORIAL

UTILIZACIÓN DE LA METODOLOGÍA MIXTA DE INVESTIGACIÓN EN SI: EL CASO DEL ESTUDIO DE LA CULTURA OCUPACIONAL DE SISTEMAS

Indira R. Guzmán

ESTUDIO EXPLORATORIO DE LOS DEPARTAMENTOS DE TECNOLOGÍAS DE INFORMACIÓN

Juan Manuel Gómez Reynoso,

Carlos Alejandro Torres Quiñones y

Javier Santiago Cortes López

FACTORES DECISIVOS PARA EL ÉXITO DE LA CAPACITACIÓN CORPORATIVA CON BASE EN LA WEB EN BRASIL: UNA INVESTIGACIÓN EXPLORATORIA MULTICASO

Luiz Antonio Joia y

Mário Figueiredo Costa

POLÍTICA EDITORIAL 
Editor-in-Chief: Carlos Ferran Penn State Great Valley Pennsylvania State University 30 E Swedesford Road Malvern, PA 19355 U.S.A. cferran@psu.edu Editorial Board:

Carlos Dorantes Tecnológico de Monterrey, México cdorante@itesm.mx

Carlos Ferran Pennsylvania State University, USA cferran@psu.edu Martha Garcia-Murillo Syracuse University, USA mgarciam@syr.edu

David Montesinos INCAE, Costa Rica Montesin@mail.incae.ac.cr

Carlos J. Navarrete California State Polytechnic University, USA cjnavarrete@csupomona.edu

James B. Pick University of Redlands, USA James_Pick@redlands.edu

Guillermo Rodríguez-Abitia Tecnológico de Monterrey, Campus Estado de México grdrz@itesm.mx

Martin Santana ESAN, Peru MSantana@esan.edu.pe

Volume 1 Number 1, 2008 (C) 2008 RELCASI Cover: Maria Elena Repiso ISSN 1937-8823 (on-line) ISSN 1937-8831 www.relcasi.org

\section{Revista Latinoamericana y del Caribe de la Asociación de Sistemas de Información RELCASI}

TABLE OF CONTENT

Volume 1 Number 1, 2008

EDITORIAL INTRODUCTION

USINGMIXEDMETHODOLOGYININFORMATIONSYSTEMS: THE CASE OF THE STUDY OF THE IT OCCUPATIONAL CULTURE 11 Indira R. Guzmán

ANEXPLORATORYSTUDYOFTHEINFORMATIONTECHNOLOGY DEPARTMENTS

Juan Manuel Gómez Reynoso,

Carlos Alejandro Torres Quiñones y

Javier Santiago Cortes López

CRITICAL SUCCESS FACTORS IN WEB-BASED CORPORATE TRAINING IN BRAZIL: AN EXPLORATORY MULTI-CASE INVESTIGATION

Luiz Antonio Joia y

Mário Figueiredo Costa

EDITORIAL POLICY

CALL FOR ARTICLES 


\title{
Utilización de la Metodología Mixta de Investigación en Sistemas de Información: El caso del estudio de la Cultura Ocupacional de Sistemas
}

\section{Using Mixed Methodology in Information Systems: The case of the study of the IT occupational culture}

\author{
Indira R. Guzmán \\ TUI University, Universidad Católica Boliviana, iguzmán@tuiu.edu
}

\section{RESUMEN}

El uso de la metodología mixta secuencial tiene poca popularidad en el área de sistemas de la información (SI). Este documento presenta inicialmente una evaluación bibliométrica del uso de la metodología mixta en publicaciones en SI. Luego se presenta un resumen de las características de metodología de investigación mixta, más un análisis de las ventajas y desventajas basándose en la revisión de la literatura. Finalmente, se presenta un estudio relacionado a la adaptación de principiantes a la cultura ocupacional en SI como un ejemplo del uso de la metodología mixta secuencial. El presente artículo contribuye a un mejor entendimiento del uso de la metodología mixta en el campo de sistemas de información. En la investigación de ejemplo, se utilizaron primero grupos focales y entrevistas semi-estructuradas como técnicas cualitativas iniciales. Posteriormente, el análisis de estos pasos cualitativos contribuyó al desarrollo de un instrumento estructurado y cuantitativo, es decir un cuestionario. Este enfoque permitió realizar un estudio más consistente agregando precisión a los resultados y validez al cuestionario cuantitativo final que puede, posteriormente, ser utilizado y replicado en el futuro. La metodología de investigación mixta es una metodología eficaz para el estudio de temas o enfoques nuevos en SI. El estudio de la cultura ocupacional en sistemas es presentado en este estudio como un ejemplo de aplicación de metodología mixta. Los resultados finales de este estudio basado en metodología mixta indicaron que la adaptación a la cultura ocupacional influye en el compromiso ocupacional en el área de sistemas de información.

Palabras Claves: Metodología mixta, Cultura Ocupacional en Sistemas de Información, Grupos Focales, Entrevista, Cuestionario, Compromiso Ocupacional 


\section{ABSTRACT}

The use of sequential mixed methodology has not been very popular in the Information Systems (IS) literature. The present study offers an initial bibliometric evaluation of the use of mixed methodology in IS publications; then, a summary of the characteristics of mixed methodology and the advantages and disadvantages of such methodological approach as presented in the literature. Finally, the development of a research study related to the adaptation of newcomers to the occupational culture in information technology occupations is presented as a sample to show the procedures of a sequential mixed methodology approach. The present study contributes to the understanding of the process of using mixed methodology in the IS. The research study sample included first qualitative elicitation techniques: focus groups and interviews. Then, the analysis of the qualitative phase served as the basis for the development and implementation of a quantitative survey. This mixed approach adds precision to the findings as well as improves the ability to replicate and expand this type of study in IS by re-using the survey instrument. The sample research shows that mixed methodology is an effective methodological approach for the study of issues that have not been addressed before in the IS literature. Specifically, the study of occupational culture conducted using mixed methodology provided empirical evidence that adaptation to the cultural characteristics influences occupational commitment in the information technology field.

Keywords: Mixed methodology, Occupational Culture of IS/IT personnel, Focus Groups, Interviews, Survey, Occupational Commitment, IT Workforce.

\section{INTRODUCCIÓN}

El uso de la metodología mixta secuencial no ha sido muy popular en la literatura de sistemas de información (SI). El propósito de este documento es en primer lugar presentar una evaluación de las aplicaciones de metodología mixta de investigación en la literatura de SI. En segundo lugar identificar las características, ventajas y desventajas de la metodología mixta de la investigación (MMI) de acuerdo a la revisión de la literatura y finalmente, ejemplificar el uso de la metodología mixta en el estudio de un tema nuevo en SI como es el caso de la cultura ocupacional en carreras de SI y la adaptación a esta cultura ocupacional de nuevos integrantes. En consecuencia, las preguntas de investigación de este estudio son las siguientes:

- Pregunta de Investigación 1: ¿Cual ha sido el uso de la metodología mixta de investigación en el área de sistemas de la información?

- Pregunta de Investigación 2: ¿Como se puede utilizar metodología mixta de investigación en el estudio de la cultura ocupacional en sistemas? 
De acuerdo a Mingers (2001), el concepto de "metodología" se refiere a un sistema estructurado de pautas o de actividades para asistir la generación de resultados de investigación válidos y confiables. Este sistema consiste a menudo en el uso de ciertas técnicas que deben ser utilizadas al mismo tiempo y con un mismo objetivo (Mingers, 2001). Los estudios de metodología mixta sin embargo, se refieren a la combinación de enfoques cualitativos y cuantitativos en la metodología de la investigación en un solo estudio en particular o en un estudio multifacético (Tashakkori y Teddlie, 1998) que además incluyen suposiciones filosóficas (Creswell and Clark, 2007). El propósito de este escrito, es enfocarse en SI y por eso a continuación se presenta una análisis del uso de metodología mixta en SI.

\section{METODOLOGÍA MIXTA EN EL ÁREA DE SISTEMAS DE LA INFORMACIÓN (SI)}

Investigadores en el área de sistemas de información (SI) han venido utilizando una gran variedad de metodologías de investigación tales como el modelado analítico, cuestionarios, casos de estudio, experimentos de laboratorio, cuasi-experimentos, simulaciones, etnografía, teorización anclada e investigación aplicada (Chua et al., 2003). Sin embargo, no existe una evaluación cuantitativa de las preferencias metodológicas en el área de sistemas y mucho menos una evaluación del uso de metodología mixta en particular. Para evaluar cómo la metodología mixta se ha utilizado en la bibliografía del área SI, se realizo un análisis bibliométrico del uso del "metodología mixta", incluyendo además el análisis biobliométrico de "casos de estudio" y "cuestionarios". La búsqueda se realizo en tres conocidas y prestigiosas publicaciones de SI (como en Larson y Neely, 2000): Management Information Systems Quarterly (MISQ), Information Systems Research (ISR) y Communications of the Association of Computing Machinery (CACM). El propósito de esta búsqueda fue encontrar un número aproximado de publicaciones en la literatura clasificada de acuerdo al método mencionado (análisis bibliométrico) y posteriormente comparar los números obtenidos y poder así demostrar las preferencias metodológicas dentro la bibliografía de SI.

El análisis bibliométrico es una técnica analítica cuantitativa para estudiar publicaciones tal cual se reflejan en la bibliografía. Es un método general que permite que la actividad en publicaciones sobre un tema específico pueda ser explicada, interpretada, o entendida de una manera cuantitativa (White y McCain 1989). El método considera los objetos, agentes, acontecimientos, productos, y contextos de la actividad en publicaciones tal que las entidades se pueden contar, medir o cuantificar (Borgman y Furner 2002). El propósito principal de utilizar esta técnica ha sido evaluar y contar el número de publicaciones que mencionaron explícitamente "metodología mixta" dentro de un cierto plazo de tiempo y según lo representado por las búsquedas de bases de datos bibliográficas. El análisis 
bibliométrico se puede también utilizar para estudiar la aparición de ediciones y de tendencias. Se desarrollo la búsqueda de criterios para las tres reconocidas publicaciones en el área usando el "número único identificador" o "ProQuest Media Identifier" (pmid) en la fuente de datos. La fuente de datos utilizada en esta búsqueda fue la base de datos global ABI/INFORM de la compañía ProQuest, un recurso para encontrar resúmenes y artículos completos en línea. ABI/INFORM consiste en un número de bases de datos, cada uno con diversa cobertura, que contiene información fidedigna (Watson-Manheim, Chudoba, y Crowston 2002). Esta base de datos incluye las tres publicaciones mencionadas con un promedio de 19 años de solamente resúmenes y 15 años de texto completo de las publicaciones. Además, se identificó un sistema de palabras claves de búsqueda a alternativas para referirse a la metodología mixta como criterios de búsqueda en Ingles tales como: "mixed methodology", "mixed-method", y "mixed method". Este es un ejemplo del código utilizado en la base de datos:

\section{"mixed methodology OR mixed-method OR mixed method AND (pmid(15436) OR pmid(11896) OR pmid(17752))"}

donde pmid(15436) es el código de MISQ, pmid(11896) es ISR, y pmid(17752) es CACM. Se obtuvieron los siguientes resultados:

Tabla 1. Frecuencia del uso de metodologías de investigación en SI

\begin{tabular}{|l|r|r|r|r|}
\hline \multicolumn{1}{|c|}{ Palabras mencionadas } & MISQ & ISR & CACM & Total \\
\hline Cuestionario (Survey) & 239 & 100 & 353 & 692 \\
\hline Casos de Estudio (Case study) & 81 & 10 & 130 & 221 \\
\hline Metodología Mixta (Mixed method) & 1 & 2 & 0 & 3 \\
\hline
\end{tabular}

Los resultados de esta revisión demuestran que los métodos mixtos como tal no fueron mencionados mayormente dentro de estas publicaciones y sólo tres publicaciones los mencionan explícitamente. Este número es muy bajo comparado a 692 artículos que mencionan "cuestionario" y a 221 que mencionen "estudio de caso". Se debe notar que la cuenta realizada en esta sección no incluye estudios que habrían incluido otros nombres designados a posibles tipos de metodología mixta. Creswell y Clark (2007) indican que en los últimos 50 años los autores han utilizado nombres tales como multimétodo, estudios integrados, combinados, híbridos, triangulación, etc. (en sus traducciones al Ingles) refiriéndose frecuentemente a la combinación de métodos, lo cual ha dificultado la búsqueda de publicaciones que utilicen este enfoque metodológico. Por este motivo, es muy probable que exista una diferencia en los resultados obtenidos en esta búsqueda bibliométrica. Sin embargo, nuestro resultado es corroborado con estudios anteriores como el de Orlikowski y Baroudi (1991) quienes encontraron que entre 1983 y 1987, 97\% de los artículos de la investigación en el área de sistemas, utilizaron un marco positivista basado sobre todo en técnicas cuantitativas. Posteriormente y partiendo de 
un cambio de política en la editorial de MISQ, en una encuesta sobre la literatura, Nandhakumar y Jones (1997) encontraron que $16 \%$ de las publicaciones utilizaron un enfoque ampliamente interpretativo con un uso regular de los casos de estudio. Estos resultados más los resultados del análisis bibliométrico presentados en esta sección, sugieren que la metodología mixta como tal, ha sido utilizada en SI más bien con muy poca frecuencia.

Es probable que el limitado uso de metodología mixta en IS se deba a la tendencia lógica que los autores del área han seguido. Un acontecimiento histórico puede explicar lo sucedido: los primeros trabajos de investigación en SI fueron inicialmente publicados en el área de administración de empresas donde las técnicas de cuestionarios y casos de estudio son parte de los estándares normativos de revisores con ese dominio de conocimiento (Straub et al. 1994). En el tiempo en que se publicaron los primeros estudios de sistemas, tales como Mason y Mitroff, 1973; Ives, Hamilton y Davis, et al 1980; Keen, et al 1980, la principal publicación académica que incluyo este tipo de artículos fue la revista "management science", la cual tiene como objetivo publicar investigaciones científicas sobre los problemas, intereses y preocupaciones de los administradores de negocios ${ }^{1}$. Posteriormente, los autores habrían continuado con esa tendencia de utilizar principalmente cuestionarios y casos de estudio (Orlikowski y Baroudi, 1991). Otro hecho que habría influenciado las preferencias metodológica en SI esta relacionado a que los estudios en esta área provienen y tienen un importante nexo con una diversidad de áreas y disciplinas clásicas como psicología, economía, sociología, matemáticas, lingüística, semiótica, etc. (Mingers, 2001) las cuales no necesariamente han promovido metodología mixta como tal.

El propósito de este estudio es contribuir a la comprensión del uso de la metodología mixta en SI para promover su uso como una posible alternativa metodológica que ayude al estudio y entendimiento de nuevos fenómenos en SI en general y aquellos en el mundo de habla hispana en particular. A continuación, se presenta una revisión de la literatura en SI acerca del uso de metodología mixta de investigación para entender las características, ventajas y desventadas de este enfoque metodológico. Posteriormente se presenta el caso de la cultura ocupacional de sistemas como ejemplo del uso de metodología mixta.

\section{REVISIÓN DE LA LITERATURA EN LA METODOLOGÍA MIXTA}

La metodología mixta de la investigación se utiliza a menudo para aumentar la robustez de los resultados y obtener una gama más amplia de participantes. Greene, Caracelli y Graham (1989) revisaron 57 estudios mixtos en los 80 y enumeraron cinco propósitos de este tipo de estudios: a)

\footnotetext{
${ }^{1}$ http://www.informs.org/site/ManSci/
} 
Triangulación: búsqueda de la convergencia y la corroboración de los resultados sobre un mismo fenómeno; b) Complementario: que examina las diferentes facetas de un fenómeno, búsqueda de elaboración, ilustración, mejora, y aclaración de las conclusiones; c) Iniciación: el descubrimiento de paradojas, contradicciones, nuevas perspectivas; d) Desarrollo: utilizando diversos métodos de forma secuencial, de manera que los resultados del primer método de informar el uso del segundo método, y e) Expansión: métodos mixtos añadiendo amplitud y alcance a un proyecto.

Onwuegbuzie y Leech (2004) sostienen que la metodología de la investigación mixta proporciona a los investigadores la oportunidad de combinar los niveles macro y micro de un estudio, pudiendo caracterizarse por tener uno o más de los fines anteriormente mencionados. Los autores identificaron cuatro retos a los que los investigadores se enfrentan a la hora de emprender métodos mixtos de investigación: Representación, legitimidad y validez, integración de datos entre diferentes métodos, y las políticas de aceptación de esta clase de resultados.

Representación: se refiere a la dificultad en la captura y representación de experiencias vividas, ya sea específicamente mediante palabras/texto o mediante números. Este problema es heredado de las metodologías puramente cuantitativas o cualitativas.

Legitimidad o validez: se refiere a la dificultades de obtener resultados y/o hacer inferencias que sean creíbles, confiables, transferibles, y/o confirmables. Este desafío incluye problemas de validez relacionados a la medición (por ejemplo, validez del instrumento, y contenido) (Shadish, Cook, y Campbell, 2001) y validez relacionada al diseño relacionados con la validez (por ejemplo, interna y externa) en el contexto cuantitativo, y la polémica relacionada a legitimidad y la transferibilidad en el contexto cualitativo.

Integración entre métodos: se refiere a la cuestión de si los investigadores pueden triangular, ampliar, comparar o consolidar grandes cantidades de datos cuantitativos recolectados de manera aleatoria, con datos cualitativos que proceden de grupos pequeños seleccionados por conveniencia. Por ejemplo, ¿cuánto peso se debe dar a los datos cualitativos frente a los datos cuantitativos?

Políticas: se refiere a las tensiones que a menudo se producen cuando se combinan enfoques cualitativos y cuantitativos, tales como las contradicciones y paradojas derivadas de la comparación, y la dificultad de persuadir a la audiencia academica acerca del valor de las conclusiones derivadas de las dos fases de un estudio. Esto es especialmente dificultoso cuando dos paradigmas epistemológicos se recogen en un mismo estudio de investigación (Leininger, 1994). 
Finalmente, de acuerdo a Tashakkori and Teddlie (1998), el método mixto es especialmente útil en temas de investigación que no se han estudiado antes. A continuación, se presenta un ejemplo del uso de metodología mixta sobre el estudio de la cultura ocupacional en SI a manera de ilustración de las características de la metodología mixta de investigación.

\section{UTILIZANDO METODOLOGÍA EN EL ESTUDIO DE LA CULTURA OCUPACIONAL EN SISTEMAS}

Este presente estudio utilizó un enfoque de metodología de investigación mixta (Tashakkori y Teddlie, 1998) para investigar el proceso de ajuste o adaptación y las preferencias individuales de los recién llegados a la cultura ocupacional en SI y las diferencias que podrían existir entre estudiantes de ambos géneros: masculino y femenino. Este estudio utilizo metodología mixta de la investigación combinando enfoques cualitativos y cuantitativos (en la colección y el análisis de datos) en un solo estudio de manera secuencial. Según Tashakkori y Teddlie (1989), en diseños secuénciales de metodología mixta, el investigador conduce una fase cualitativa de un estudio y luego una fase cuantitativa separada, o viceversa. En este particular estudio presentado como ejemplo, se ha utilizado un enfoque secuencial mixto con los siguientes propósitos: (a) estudiar un fenómeno nuevo (la adaptación a la cultura ocupacional) y (b) desarrollar un cuestionario (en la parte cuantitativa). La primera etapa que fue cualitativa consistió en el uso de grupos focales y entrevistas semiestructuradas. En la segunda etapa cuantitativa se construyo un cuestionario con el que se recolecto datos mediante Internet. La parte cuantitativa se basó en los resultados obtenidos en la fase 1 como se indica en el grafico 1 de una manera secuencial. Las dos fases fueron diseñadas para mejorar la validez del material de investigación y obtener resultados más robustos y validos.

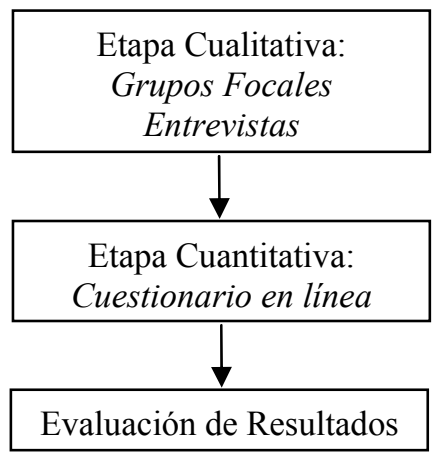

Figura 1. Metodología secuencial mixta

A continuación, se presentan las definiciones y descripción detallada de los conceptos relacionados al tema de la cultura ocupacional en sistemas y de la forma en que el estudio fue conducido metodológicamente. 


\section{EI Estudio de la Cultura Ocupacional en SI}

El concepto de "cultura" en general es una variable muy importante que puede explicar cómo grupos sociales interactúan recíprocamente con las tecnologías de la información y comunicación (TIC). Por otro lado, un estudio reciente sobre las investigaciones relacionadas a aspectos culturales en SI, revela que existen importantes oportunidades de investigación para definir valores, actitudes y adaptación cultural en SI (Leidner y Kayworth, 2006). Cultura se puede definir como las filosofías compartidas, ideologías, valores, creencias, expectativas, actitudes, y normas que miembros de un grupo tienen en común dentro de una comunidad (Hofstede, 1997; Trice, 1993). Harrison Trice (1993) identificó las sub-culturas ocupacionales dentro de organizaciones, refiriéndose a racimos o subdivisiones de ideologías, creencias, formas culturales, y prácticas de los individuos que forman parte de una comunidad ocupacional o laboral. Estos valores se originan en las experiencias educativas y personales compartidas dentro de la comunidad ocupacional. Las organizaciones tienen por lo tanto muchos subgrupos basados en grupos ocupacionales que se desarrollan dentro de la organización y que conforman sus propios culturas. Uno de esos subgrupos es conformado por la gente que trabaja en SI. El estudio de la cultura ocupacional en SI es un tema novedoso que no ha sido anteriormente investigado y desarrollado bajo un marco teórico como el de Trice (1993) y Douglas (1982). Este documento presenta los detalles metodológicos del estudio realizado en el tema.

Los primeros estudios sobre la cultura ocupacional en SI que se han reportado empíricamente fueron puramente cualitativos basados en entrevistas y casos de estudio (Guzmán y otros., 2004; Gerulat, 2002; Ramachandran y Rao, 2006). Aplicando los conceptos desarrollados por Trice (1993), los resultados de esos estudios cualitativos indican que efectivamente existe una cultura ocupacional propia en el área SI (Guzmán, Stam y Stanton, 2008). De acuerdo a estos resultados, la cultura ocupacional de SI se caracteriza por el alto aprecio del conocimiento técnico, las extremas e inusuales demandas de tiempo en la profesión, la sensación de superioridad en relación a los usuarios de sistemas, una carencia típica de las reglas de trabajo formales, el uso frecuente de la tecnología fuera de horas de trabajo y finalmente la presencia de formas culturales tales como el uso de la jerga técnica en la conversación y el reconocimiento de la existencia de estereotipos sociales como "geek" y "nerd". Sin embargo, no existía evidencia empírica alguna sobre la adaptación o aculturación de los nuevos integrantes de esta fuerza laboral.

A continuación se presenta un análisis detallado de los aspectos metodológicos que se tomaron en cuenta en el estudio de la aculturación o proceso de adaptación de nuevos individuos a la cultura ocupacional en SI. Primero, se describe el uso de los grupos focales utilizados para entender 
como los participantes se familiarizaron y socializaron con la cultura ocupacional en SI. Después se describen las entrevistas que fueron conducidas con estudiantes universitarios con experiencia laboral en SI. Finalmente, se describe el uso del cuestionario en línea.

\section{Grupos Focales - Focus Groups}

Para obtener una comprensión general del proceso de adaptación a la cultura ocupacional y para estimular la elaboración explícita de opiniones, motivos y razones sobre el tema desde el propio punto de vista de los participantes, elegimos conducir nuestra colección inicial de datos bajo la forma de reuniones semi-estructuradas y mediante la interacción de grupos. El acceso a este tipo de datos seria reducido sin la interacción de un grupo (Morgan, 1997; Pini, 2002). El estudio ocurrió en dos instituciones académicas en Nueva York, una universidad privada y una universidad estatal. Los datos fueron recogidos durante aproximadamente 5 meses desde octubre del 2004 hasta febrero del 2005.

Los grupos focales fueron planificados y evaluados mediante un proceso muy cuidadoso de revisión de la bibliografía en el tema de aculturación y continuas discusiones del grupo de investigadores constituido por la autora y otros 5 investigadores (un profesor, 4 estudiantes). El protocolo fue revisado y probado en el grupo antes de ser utilizado con los participantes para asegurar un claro entendimiento de las preguntas como así también practicar el protocolo. Desarrollamos el protocolo tomando el cuenta la bibliografía, las preguntas de investigación del estudio y las experiencias adquiridas en el proceso durante y después de cada grupo focal. En conformidad con la práctica común de investigación cualitativa (Denzin y Lincoln, 1994; Janesick, 1998) se desarrollaron las preguntas para expandir nuestro entendimiento del tema "adaptación a la cultura ocupacional en SI". La base del protocolo del grupo focal cubrió áreas tales como percepciones sobre las razones de elegir carreras en SI, opiniones generales sobre la carrera y las actividades cotidianas, estereotipos conocidos en la sociedad y posibles razones para formar o no formar parte de este grupo ocupacional. En general, se observo una clara diferencia en las percepciones de quienes tenían previa experiencia laboral y quines carecían de ella. Los participantes que no tenían experiencia laboral no eran capaces de contribuir activamente en la discusión. Los investigadores llegaron a esta conclusión unánimemente y de manera progresiva durante las reuniones en las que se analizaba cada grupo focal.

Se condujeron un total de nueve grupos focales con un promedio de 7 participantes en cada discusión de grupo. Cada grupo focal contaba con la presencia de un moderador, una persona encargada de grabar la sesión en video y una persona encargada de tomar apuntes sobre las reacciones. Todos los participantes invitados eran estudiantes de carreras de sistemas. Seleccionamos este tipo de participantes porque además de ser un grupo conveniente y de fácil acceso, ellos/as pueden recordar más fácilmente las 
razones por las que eligieron esta carrera ya que sus decisiones fueron tomadas más recientemente en comparación con aquellos que han estado en la ocupación por un tiempo más largo. Cada discusión duro 55 minutos en promedio. Dos de los grupos focales fueron organizados solamente con participantes del género femenino, y una moderadora del sexo femenino, para proporcionar un ambiente más homogéneo y permitir a las participantes discutir y presentar libremente sus opiniones. Esto se hizo porque uno de los objetivos del estudio era saber si las mujeres tenían otro tipo de percepción acerca de las carreras de SI y su cultura. Los otros 7 grupos tenían estudiantes de ambos sexos. Las discusiones de los 9 grupos focales fueron grabadas en video y posteriormente transcritas. En las transcripciones no se incluyeron nombres de los participantes y se diferencio la participación de mujeres con una $\mathrm{F}$ para participantes femeninas y $\mathrm{M}$ para los participantes masculinos.

El resultado o conclusión más importante a la que se llego luego del análisis de los grupos focales, indica que la gente mejor informada acerca de las características culturales de la ocupación son solamente aquellos/as que obtuvieron algún tipo de experiencia laboral en SI. Los estudiantes sin experiencia profesional no pudieron articular las normas, valores y formas culturales de la ocupación porque no estaban al tanto de ellas. Por ejemplo, aquellos que no tuvieron experiencia laboral no podían articular acerca de su interacción con los usuarios o su relación con colegas de SI en el trabajo, no podían decirnos sobre los desafíos regulares de la ocupación tales como la constante re-educación para mantenerse actualizados/as con la tecnología necesaria. Debido a este importante resultado, en la siguiente etapa del estudio se entrevistaron solamente a personas que contaban con experiencia laboral en SI y que por lo tanto estaban familiarizados con la cultura ocupacional tal cual fue definida en la literatura.

\section{Entrevistas}

Posteriormente, en esta fase del estudio, utilizamos entrevistas semiestructuradas con un propósito también exploratorio y para evaluar las características de la adaptación a la cultura ocupacional de los recién llegados o nuevos integrantes de la ocupación. Los participantes fueron 27 estudiantes de tres escuelas en dos instituciones académicas de los E.E.U.U. en programas de tales como informática, tecnología y gerencia de información, e ingeniería de sistemas. Todos los estudiantes recibieron una entrada de cine como incentivo para participar en las entrevistas. Entrevistamos a 19 participantes del sexo masculino (el 70\%) y a 8 participantes del sexo femenino (del 30\%). Se reclutaron a los participantes invitándolos durante sus clases, quienes llenaron un formulario indicando su la experiencia de trabajo, su dirección electrónica y teléfono para ser contactados en caso de que estuvieran interesados en participar en el estudio. 
Dirigidos por el marco teorético de Trice (1993) y Douglas (1982) desarrollamos a guía semi-estructurada de entrevista que fue utilizada para organizar la colección de datos durante la entrevista. Elegimos las entrevistas semi-estructuradas porque son "razonablemente objetivas mientras que todavía permiten una comprensión cuidadosa de las opiniones del participante y las razones detrás de ellas" (Borg y Gall, 1989, p. 452). Nuestro protocolo fue desarrollado de tal manera que pudiera ayudarnos a entender opiniones de los estudiantes sobre las características de la cultura ocupacional y su adaptación. El protocolo fue ajustado a formato en una sola página para fácil manipulación puesto que muchas veces es necesario cambiar el orden de las preguntas dependiendo de la línea de la conversación con el/la participante. La naturaleza flexible de las preguntas permitió a los participantes la libertad de hablar sobre sus creencias y percepciones o impresiones individuales así como también sobre los aspectos positivos y negativos de la ocupación. Una vez que se transcribieron las entrevistas, la recopilación de los datos ocupó cerca de 162 páginas de texto (aproximadamente 71.350 palabras). Se dejaron de conducir entrevistas cuando todos los investigadores estuvieron de acuerdo en que ya no se obtenía ninguna nueva información de los participantes acerca del tema (utilizando las mismas preguntas), es decir, cuando se alcanzó una saturación de los datos (Miles y Huberman, 1994).

Éste es un ejemplo de las preguntas usadas en la entrevista:

- Perspectivas personales sobre la ocupación:

$\circ$ ¿Por qué elegiste esta carrera/profesión?

○ ¿Cómo es esta ocupación comparada a otras ocupaciones?

$\circ$ ¿Te gusta o no el nivel de la demanda?

$\circ$ ¿Cuáles son algunos de los estereotipos de la gente de sistemas?

$\circ$ ¿Qué tipos de problemas tiene la gente en esta ocupación/profesión?

Como se indicó anteriormente y basándonos en la teoría de Trice, una de las características de la cultura ocupacional es el valor del conocimiento técnico (conocimiento esotérico). La mayor parte de nuestros participantes indicaron que se sienten satisfechos cuando pueden solucionar problemas y que les gusta el desafío. Otras respuestas incluyeron el gusto de probar nuevas tecnologías, tomar clases/certificaciones técnicas y estar en línea (en Internet) la mayor parte del tiempo (inclusive en sus horas libres). También proporcionaron una lista extensiva de estereotipos de la gente en SI. Estas percepciones también son expresadas en las siguientes cuotas:

"En sistemas, la gente tiene conocimiento especializado y ellos saben que tienen ese conocimiento especializado."

"Me gusta tener que aprender nuevas cosas constantemente. Pienso que eso incomoda probablemente 
a alguna gente y quizá es la razón porque la gente no escoge estas carreras [en SI]. Lo que pasa, es que este campo está cambiando constantemente!"

"Pienso que el trabajo es desafiante, hay muchos problemas que solucionar"

"Me siento horas a leer los artículos sobre tecnología. Una razón es el miedo, porque si no lo haces, te quedas obsoleto/a."

El cuestionario para la parte cuantitativa fue desarrollado usando el vocabulario expresado en la fase cualitativa. Las opiniones expresadas en las entrevistas fueron discutidas $\mathrm{y}$ analizadas por el grupo de investigación. Basados en las expresiones más comunes de nuestros participantes, se desarrollaron escalas para cada una de las dimensiones de la cultura ocupacional. A continuación, se presenta como ejemplo una escala de preguntas sobre "conocimiento esotérico" utilizada en la fase cuantitativa del estudio:

Instrucciones: ¿Qué te gusta o disgusta sobre los desafíos en el área de sistemas?

Indica por favor el grado de preferencia o discrepancia con las declaraciones siguientes. (1-en completo desacuerdo a 6-Completamente de acuerdo).

\section{Escala del conocimiento esotérico}

Preguntas

1. Me gusta aprender cómo solucionar problemas de tecnologías de la información.

2. Yo disfruto adquiriendo experiencia practica con tecnologías de la información.

3. Realmente me gusta aprender lo nuevo sobre tecnologías de la información.

4. Actualizarme en lo último en tecnología es divertido para mí.

\section{Cuestionario}

Finalmente, utilizamos los resultados de la entrevista de la fase 1 para formular siete escalas experimentales que correspondían a las siete dimensiones de la cultura ocupacional. Para cada escala, se llevo a cabo un proceso iterativo para la generación de preguntas utilizando primero participantes voluntarios en ejercicios de prueba. Se hicieron cuatro iteraciones de este proceso para tener las mejores preguntas que tendrían el mismo significado para los participantes. Después, se condujo un estudio piloto para validar el cuestionario con aproximadamente 50 participantes y 
poder así determinar características psicometrías del instrumento. Nuestro cuestionario final fue utilizado con 215 participantes. Las escalas fueron validadas usando las herramientas estadísticas tales como análisis factorial y de Alfa de Cronbach o confiabilidad. Nuestra escala sobre la adaptación a la cultura ocupacional en SI tuvo 34 preguntas y una estimación de confiabilidad mayor a .70 en todos los casos, tal como se muestra en la tabla 2. Luego de haber creado la nueva escala para adaptación cultural, se identifico una variable dependiente anteriormente utilizada bibliografía académica llamada "compromiso ocupacional" estudiada y definida por Meyer, Allen y Smith (1993) y Meyer, Becker y Vandenberghe (2004). Esta escala para evaluar compromiso ocupacional en su versión en Ingles fue utilizada y validada por Meyer y otros. En este estudio, se utilizo la escala adaptada al contexto de SI. $^{2}$

Tabla 2. Dimensiones de la cultura ocupacional en SI

\begin{tabular}{|l|l|c|c|}
\hline \multicolumn{1}{|c|}{ Escala } & \multicolumn{1}{|c|}{ Definición } & $\begin{array}{c}\text { Número } \\
\text { de } \\
\text { Preguntas }\end{array}$ & $\begin{array}{c}\text { Confiabilidad } \\
\text { (Alfa de } \\
\text { Cronbach) }\end{array}$ \\
\hline $\begin{array}{l}\text { Estereotipos } \\
\text { Geek/Nerd }\end{array}$ & $\begin{array}{l}\text { Estigmatizados como nerds o geeks; } \\
\text { historias compartidas sobre errores de } \\
\text { los usuarios. }\end{array}$ & 6 & .70 \\
\hline $\begin{array}{l}\text { Jerga } \\
\text { Idiomática }\end{array}$ & Constante uso de terminología técnica & 3 & .74 \\
\hline $\begin{array}{l}\text { Auto- } \\
\text { confianza }\end{array}$ & $\begin{array}{l}\text { Confianza de poder satisfacer las } \\
\text { demandas de la ocupación }\end{array}$ & 8 & .92 \\
\hline $\begin{array}{l}\text { Conocimiento } \\
\text { Esotérico }\end{array}$ & $\begin{array}{l}\text { Orgullo de tener conocimiento, } \\
\text { habilidades y capacidades técnicas } \\
\text { únicas; Gran valor del conocimiento } \\
\text { técnico }\end{array}$ & 6 & .92 \\
\hline Desafíos & $\begin{array}{l}\text { Gran apreciación del desafío, de } \\
\text { ocuparse de tareas difíciles, largas } \\
\text { horas, cambios constante, y de } \\
\text { necesidad de ser autodidacta }\end{array}$ & 5 & .72 \\
\hline $\begin{array}{l}\text { Integración } \\
\text { de TI }\end{array}$ & $\begin{array}{l}\text { La integración del uso de TI fuera del } \\
\text { trabajo y en el tiempo libre. }\end{array}$ & 6 & .82 \\
\hline
\end{tabular}

Los principales resultados obtenidos del enfoque mixto, incluyendo la fase cuantitativa en la que se realizaron análisis estadístico con SPSS, son los siguientes $^{3}$ :

- La adaptación a las características culturales de la ocupación es un buen antecedente (puede predecir) el compromiso ocupacional en SI.

${ }^{2}$ La versión completa del instrumento cuestionario está disponible en Guzmán (2006)

${ }^{3}$ Los resultados estadísticos del estudio pueden se encontrados en Guzmán (2006) y Guzmán y Stanton (2008). 
- Existen diferencias de adaptación/aculturación en términos de género, grupo étnico y tiempo de experiencia profesional.

- Los nuevos profesionales en SI absorben y se familiarizan con la cultura ocupacional principalmente durante experiencias de trabajo.

- La gente con más experiencia profesional en SI presentó opiniones más positivas hacia las características de la cultura ocupacional y por lo tanto, mejor adaptación.

- Los varones demostraron mayor auto-confianza en relación a las demandas de la ocupación y que además integran más extensivamente la tecnología en sus horas libres en comparación a las participantes femeninas.

Es importante mencionar que los tres últimos resultados de la lista fueron inicialmente descubiertos en la etapa cualitativa, pero se confirmaron estadísticamente en la etapa cuantitativa. Por ejemplo, la variable de tiempo de experiencia laboral dio resultados significativos estadísticamente pero este ya había sido un factor mencionado en el análisis de la etapa cualitativa.

\section{CONCLUSIONES}

La metodología de investigación mixta es utilizada fundamentalmente para aumentar robustez a los resultados y obtener una gama de enfoques más amplia de los participantes (Bonoma, 1985). La principal premisa del uso de métodos cualitativos y cuantitativos es que esta combinación permite un mejor entendimiento del tema de investigación que el que se podría obtener mediante el uso de uno de los métodos por separado. La aplicación de metodología mixta en este estudio realmente permitió aumentar robustez a los resultados sobre la adaptación de nuevos integrantes de la profesión y además un mejor dominio del tema estudiado.

Es así que este ejemplo de investigación ha utilizado metodología mixta con el un propósito complementario y de expansión, de acuerdo a la definición de Greene, Caracelli y Graham (1989), porque el diseño de metodología mixta permitió mejorar la validez del estudio. La metodología mixta sirvió para obtener información valiosa y detallada de la etapa cualitativa y además datos estructurados significativos estadísticamente en la etapa cuantitativa.

De acuerdo a los resultados obtenidos en este estudio, podemos concluir que la metodología mixta ha sido útil en la investigación de un concepto antes nunca visto como ha sido la adaptación a la cultura ocupacional en SI. La principal ventaja del uso de metodología mixta es la profundidad con la que se puede investigar conceptos nuevos mediante la fase cualitativa sin tener que realizar un análisis exhaustivo y detallado de los datos cualitativos como podría ser el típico análisis del contenido analítico cualitativo con herramientas como las de Atlas-TI. Los datos cualitativos 
sirvieron sobretodo, para la creación de preguntas de tipo cuantitativo utilizando el lenguaje de los mismos sujetos de estudio tal y como fue utilizado en los grupos focales y en las entrevistas. El uso de grupos focales permitió a los investigadores obtener un conocimiento detallado del fenómeno de estudio abriendo la posibilidad a la creación de una serie de hipótesis, las cuales pudieron ser revisadas durante las entrevistas y posteriormente evaluadas en la etapa cuantitativa. En cada nueva entrevista, los investigadores pueden ir mejorando el tipo de preguntas e ir identificando los temas relacionados con el tema de estudio principal. El proceso cuantitativo en este estudio basado en metodología mixta fue mucho más preciso comparado con el proceso tradicional de creación de escalas cuantitativas que solamente están basadas en la bibliografía e iteraciones puramente cuantitativas. Basándonos en los retos identificados por Onwuegbuzie y Leech (2004), este estudio permitió una representación valiosa textual y numérica; legitimidad y validez con gran potencial de crecimiento ya que se facilita la replicación (otros autores pueden utilizar el instrumento); integración entre métodos a la hora de comparar los resultados cualitativos y cuantitativos como los relacionados a la integración de sistemas en el tiempo libre y la importancia del tiempo de experiencia laboral en SI y las políticas mas bien compartidas, ya que este tipo de estudios puede ser aceptado en diferentes estilos metodológicos.

Uno de los problemas del uso de metodología mixta es que puede ser costoso porque requiere una serie de recursos logísticos como la participación de investigadores entrenados, moderadores, salas donde realizar los grupos focales que sean silenciosas y adecuadas para las reuniones, grabadoras de video y audio, maquinas de trascripción, incentivos para los participantes, software y hardware para el desarrollo del cuestionario en línea, y finalmente SPSS u otra herramienta para realizar análisis estadístico.

Este estudio ha presentado un enfoque de metodología mixta y un ejemplo de su aplicación en el estudio de la adaptación de cultura ocupacional en SI. El actual estudio contribuye a la comprensión del proceso del uso de metodología mixta en el área de SI para el estudio de temas y conceptos novedosos. Por ejemplo, se podría utilizar metodología mixta en el uso de fenómenos relacionados al uso de tecnologías de la información en Latino América donde muchas áreas no han sido aun investigadas en ese contexto geográfico. La consistencia de metodología mixta agrega precisión y robustez a los resultados así como validez a cuestionarios que pueden ser posteriormente mejorados, replicados y así obtener mayor generalización.

Este tipo de estudios pueden ser un importante punto de partida en los estudios de investigación de SI. El uso de metodología mixta SI esta siendo cada vez más utilizado y promovido. En una búsqueda de presentaciones recientes en las conferencias de la Asociación de SI (AIS), 
se encontraron trabajos recientes como el de Zachariadis y Scott (2007) y Lane y Van der Vyver (2005). Adicionalmente, publicaciones recientes como la de Creswell y Clark (2007) y la creación de la nueva revista "Journal of Mixed Methods Research" de SAGE (reconocida editorial) en 2007 promueven el uso de la metodología mixta.

\section{AGRADECIMIENTOS}

Este proyecto contó con el patrocinio de la Fundación Nacional de Ciencia de los Estados Unidos (ITWF NSF Award\# 0420434). La autora agradece sinceramente a Carlos J. Navarrete, Martha Garcia-Murillo y James Pick que organizaron el "minitrack" en Español de la conferencia de la Asociación Americana de Sistemas de Información in Colorado, Estados Unidos en 2007, donde una versión inicial de este escrito fue presentada. Asimismo, la autora agradece a los revisores de la revista RELCASI por los valiosos comentarios que ayudaron a mejorar la calidad de este escrito y a Santos Miguel Galvez y Daniel Calvo-Montes por sus comentarios editoriales.

\section{BIBLIOGRAFÍA}

Allen, N. J., \& Meyer, J. P. (1993). Organizational commitment: Evidence of career stage effects? Journal of Business Research, 26(1), 49-61.

Babbitt, T. (2001). A tale of two shortages: An analysis of the IT professional and MIS faculty. Paper presented at the SIGCPR 2001, San Diego CA.

Blau, G. (1985). The measurement and prediction of career commitment. Journal of Occupational Psychology, 58, 277-288.

Blau, G. (1999). Early-Career Job Factors Influencing the Professional Commitment of Medical Technologists. Academy of Management Journal, 42(6), 687-695.

Blau, G. (2003). Testing for a four-dimensional structure of occupational commitment. Journal of Occupational and Organizational Psychology, 76, 469-488.

Bonoma, T. V. (1985). Case Research in Marketing: Opportunities, Problems, and a Process. Journal of Marketing Research, 22(2), 199208.

Borg, W. R., \& Gail, M. D. (1989). Educational research: an introduction. New York: Longman.

Borgman, C.L. and J. Furner. 2002. "Scholarly Communication and Bibliometrics." Annual Review of Information Science and Technology 36, 3-72.

Cable, D. M., \& DeRue, D. S. (2002). The convergent and discriminant validity of subjective fit perceptions. Journal of Applied Psychology, $87(5), 875$.

Cameron, D. (2001). Chefs and occupational culture in a hotel chain: A grid-group analysis. Tourism and Hospitality Research, 3(2), 103115. 
Chua C, Cao L, Cousins K and Straub DW (2003) Measuring researcher production in information systems. Journal of the AIS 3(6), 145-215.

Creswell, J. W., \& Clark, V. L. P. (2007). Designing and Conducting Mixed Methods Research. Thousand Oaks, California: SAGE Publications, Inc

Denzin, N. K., \& Lincoln, Y. S. (Eds.). (1994). Handbook of Qualitative Research. Thousand Oaks, CA: Sage.

Douglas, M. (1978). Cultural Bias. London: Royal Anthropological Institute of Great Britain and Ireland.

Douglas, M. (1982). Introduction to grid/group analysis. In M. Douglas (Ed.), Essays in the Sociology of Perception. London: Routledge.

Freidson, E. (1986). Professional Powers: A Study of the Institutionalization of Formal Knowledge. Chicago: University of Chicago Press.

Gerulat, R. W. (2002). Cultural characteristics of IT professionals: an ethnographic perspective. In E. Szewczak \& C. Snodgrass (Eds.), Managing the human side of information technology: challenges and solutions (pp. 311-342). Hershey, PA, USA: Idea Group Publishing.

Greene, J. C., Caracelli, V. J., \& Graham, W. F. (1989). Toward a conceptual framework for mixed-method evaluation designs. Educational Evaluation and Policy Analysis, 11, 255-274.

Guzmán, I. R. (2006). "As you like I.T.": Occupational Subculture and Commitment of New Information Technologists. Unpublished Dissertation, Syracuse University, Syracuse, NY.

Guzmán, I. R., Stam, K. R., \& Stanton, J. M. (2008). The Occupational Culture of IS/IT Personnel within Organizations. The Data Base for Advances in Information Systems, 39(1), 33-50.

Guzmán, I. R., Stanton, J. M., Stam, K. R., Vijayasri, V., Yamodo, I., Zakaria, N., \& Caldera, C. (2004). A Qualitative Study of the Occupational Subculture of Information Systems Employees in Organizations. Paper presented at the ACM Special Interest Group on Management Information Systems - Computer Personnel Research Conference, Tucson, Arizona.

Guzmán, I. R., Stanton, J. M., \& Eischen, D. (2006). Female Perceptions of the Information Technology Culture. In E. M. Trauth (Ed.), Encyclopedia of Gender and Information Technology: Idea Group Inc.

Guzman, I. R., \& Stanton, J. M. (2008). Women's Adaptation to the IT Culture. Women's Studies: Special Issue on Women and Technology, 37(3), 202-228.

Hansen, C. D. (1995). Occupational cultures: Whose frame are we using? The Journal for Quality and Participation, 18(3), 60-65.

Hofstede, G., Neuijen, B., Ohayv, D. D., \& Sanders, G. (1990). Measuring Organizational Cultures: A Qualitative and Quantitative Study Across Twenty Cases. Administrative Science Quarterly, 35(2), 286-316. 
Ives, B., Hamilton, S., \& Davis, G. B. (1980). A Framework for Research in Computer-Based Management Information Systems. Management Science 26(9), 910-934.

Janesick, V. J. (1998). Stretching Exercises for Qualitative Researchers. Thousand Oaks: Sage Publications.

Keen, P. G. W. (1980), MIS Research: Reference Disciplines and a Cumulative Tradition. Proceedings of the First International Conference on Information Systems, 9-18.

Lane, M., \& Van der Vyver, G. L. (2005). Partnership Quality in IT outsourcing-A Mixed Methods Review of its Measurement. Paper presented at the 16th Australasian Conference on Information Systems, Sydney, Australia.

Larsen, K. and P. Neely. 2000. Profiles of MIS Doctoral Candidates: Ideas and Reality. The DATABASE for Advances in Information Systems 31(3), 64-77.

Lee, K., Carswell, J., \& Allen, N. (2000). A meta-analitic review of Occupational Commitment: Relations with person and work-related variables. Journal of Applied Psychology, 78, 538-551.

Leidner, D. E., \& Kayworth, T. (2006). A Review of Culture in Information Systems Research: Towards a Theory of IT-Culture Conflict. MIS Quarterly, 30(2), 357-399.

Leininger, M. (1994). Evaluation Crieteria and Critique of Qualitative Research Studies. In J. M. Morse (Ed.), Critical Issues in Qualitative Research Methods. Thousand Oaks, CA: SAGE Publications.

Lincoln, Y. S., \& Guba, E. G. (1985). Naturalistic Enquiry. Beverly Hills: Sage.

Mason, R. O., \& Mitroff, I. I. (1973). A Program for Research on MIS. Management Science 19(5), 475-487.

May, T. Y.-M., Korczynski, M., \& Frenkel, S. J. (2002). Organizational and occupational commitment: Knowledge workers in large corporations. Journal of Management Studies, 39(6), 775-801.

Mencil, J. (2005). Multipotentiality In The Workplace: PersonEnvironment Fit, Occupational Outcomes, And Emotional Intelligence.Unpublished manuscript.

Meyer, J. P., \& Alien, N. J. (1991). A three-component conceptualization of organizational commitment. Human Resource Management Review, 1(1), 61-89.

Meyer, J. P., Allen, N. J., \& Smith, C. A. (1993). Commitment to organizations and occupations: Extension and Extension and Test of a Three-component Conceptualization. Journal of Applied Psychology, 78(4), 538.

Meyer, J. P., Becker, T. E., \& Vandenberghe, C. (2004). Employee Commitment and Motivation: A Conceptual Analysis and Integrative Model. Journal of Applied Psychology, 89(6), 991-1007. 
Miles, M. B., \& Huberman, M. (1994). Qualitative Data Analysis: An Expanded Sourcebook (2nd ed.). Thousand Oaks, CA: SAGE.

Mingers, J. (2001). Combining IS research methods: Towards a pluralist methodology. Information Systems Research, 12(3), 240-260.

Morgan, D. L. (1997). Focus Groups as Qualitative Research (Second ed.). London: Sage.

Nandhakumar, J. and Jones, M. (1997). Too close for comfort? Distance and engagement in interpretive information systems research. Information Systems Journal (7:2), 1997, pp. 109-131.

Onwuegbuzie, A. J., \& Leech, N. L. (2004). Enhancing the Interpretation of "Significant" Findings: The Role of Mixed Methods Research. The Qualitative Report, 9(4), 770-792.

Orlikowski, W. J., \& Baroudi, J. J. (1989). The Information Systems Profession: Myth or Reality? Office: technology and people., 4(1), 1330.

Orlikowski, W. J., \& Baroudi, J. J. (1991). Studying Information Technology in Organizations: Research Approaches and Assumptions. Information Systems Research, 2, 1-28.

Pini, B. (2002). Focus Groups, Feminist Research and Farm Women: Opportunities for empowerment in rural social research. Journal of Rural Studies, 18, 339-351.

Ramachandran, S., \& Rao, S. V. (2006). An effort towards identifying occupational culture among information systems professionals. Paper presented at the 2006 ACM SIGMIS CPR conference on computer personnel research: Forty four years of computer personnel research: achievements, challenges \& the future SIGMIS CPR '06.

Reichers, A. E. (1985). A Review and Reconceptualization of Organizational Commitment. Academy of Management. The Academy of Management Review, 10(3), 465-476.

Shadish, W. R., Cook, T. D., \& Campbell, D. T. (2001). Experimental and Quasi-experimental designs for generalized causal inferences. Berkeley: Houghton Mifflin.

Tashakkori, A., \& Teddlie, C. (1998). Mixed Methodology: Combining Qualitative and Quantitative Approaches (Vol. 46). Thousand Oaks, CA: SAGE Publications, Inc.

Tashakkori, A., \& Teddlie, C. (2003). The past and future of mixed methods research: From data triangulation to mixed model designs. In A. Tashakkori \& C. Teddlie (Eds.), Handbook of mixed methods in social and behavioral research. Thousand Oaks, CA: SAGE.

Trice, H. (1993). Occupational Subcultures in the Workplace. Ithaca, NY: ILR Press.

Trice, H. M., \& Beyer, J. M. (1984). Studying Organizational Cultures Through Rites and Ceremonials. Academy of Management. The Academy of Management Review, 9(4), 653-669. 
Watson-Manheim, M. B., Chudoba, K. M. \& Crowston, K..(2002).

"Discontinuities and continuities: a new way to understand virtual work." Information Technology \& People 15:191-203.

White, H.D. and K.W. McCain. 1989. "Bibliometrics." Annual Review of Information Science and Technology 24:119-186.

Zachariadis, M., \& Scott, S. V. (2007). Diversity in IS Research: Developing a Mixed Methodology Approach to Understanding the Business Value of Payment System Innovations in Financial Services. Paper presented at the International Conference of Information Systems, Montreal, Canada.

Indira R. Guzmán, Ph.D., es profesor asistente adjunto y coordinadora académica de sistemas de información y administración de empresas en la Universidad de TUI en California, investigador asociado de la Universidad de Syracuse en Nueva York y profesor visitante de la Universidad Católica Boliviana. Su experiencia laboral en tecnologías de la información incluye más de quince años como profesional, académico y consultor de sistemas. Sus intereses de investigación incluyen el impacto de las TIC en las organizaciones, la seguridad de la información, aprendizaje en línea y principalmente el estudio de los recursos humanos, su administración, contratación, retención y la representación femenina en sistemas. Sus publicaciones figuran en revistas académicas como The DATA BASE for Advances in Information Systems, Human Resource Management, Women's Studies, y Journal of Digital Information. La Dra. Guzmán obtuvo el Doctorado en Ciencias de la Información y Tecnología y la Maestría en Administración de la Información de la Universidad de Syracuse y la Licenciatura y Maestría en Ingeniería de Sistemas de la Universidad Politécnica de Donetsk en Ucrania. 


\section{Política Editorial}

RELCASI está principalmente dirigida a investigadores de habla hispana en el área de Administración de Sistemas de Información (MIS por sus siglas en Inglés). Los artículos son académicamente rigurosos sin sacrificar la claridad, estilo, simplicidad y contribución práctica que los hace atractivos a profesionales de la disciplina. En consecuencia, la audiencia de esta revista está compuesta no sólo por investigadores de MIS, sino también por profesionales y administradores en el área de tecnologías de información.

Todos los artículos son escritos, revisados y publicados en español; sin embargo, estos contendrán título, resumen y palabras claves en español y en inglés.

RELCASI es una publicación arbitrada que se presenta en formato impreso y en línea. La versión impresa de RELCASI está disponible a pedido (y próximamente bajo subscripción). La versión en línea se provee a través de la Asociación de Sistemas de Información. El proceso de evaluación se realizará con al menos dos examinadores. La identidad del editor asociado y de los examinadores no será del conocimiento del autor, y ni los examinadores ni el editor asociado conocerán la identidad del autor. Una ronda del proceso de evaluación tomará alrededor de 90 días.

La revista incluye principalmente artículos de investigación desarrollados con un marco teórico robusto y que incluyan una adecuada revisión de literatura. Los artículos podrán ser de investigación empírica (cualitativa o cuantitativa), conceptuales, encuestas de corrientes de investigación, o encuestas de la industria de TI en países en desarrollo. Los artículos de investigación empírica, deben proveer una amplia justificación $y$ descripción de la colección de datos, metodología y técnicas analíticas. Estudios de caso, artículos pedagógicos, revisión de libros, y debates y ensayos de opinión serán considerados pero no formarán el grueso de la publicación. Artículos con un alto contenido técnico y bajo contenido gerencial/administrativo no son recomendados y sólo serán aceptados cuando sean altamente relevantes o innovadores. Los artículos deberán tener una extensión no mayor a las 8.000 palabras. 


\section{Editorial Policy}

RELCASI is primarily directed to Spanish speaking researchers in the area of Management Information Systems (MIS). Articles will have academic rigor without sacrificing clarity, style, simplicity, and a practical contribution that will also make them attractive to practitioners. Therefore, its audience includes both academics and practitioners of MIS and IT.

Articles are written, reviewed, and published in Spanish; however, their title, abstract, and keywords will also be published in English.

RELCASI is a double-blind peer-reviewed journal that is both in-print and on-line. The print version is currently provided on-demand and we will soon have a subscription service. The on-line version is available through the Association for Information Systems. The double-blind peer-review process will involve an associate editor and a minimum of two academic peers. We aim to have a round of the review process take no more than 90 days.

The journal will primarily comprise of research articles developed with a robust theoretical framework that include an appropriate literature review. The articles could be qualitative or quantitative, conceptual, research stream surveys, or surveys that relate to IT/MIS in developing countries. Empirical research articles must include a clear, comprehensive, and concise description of the methodology, data collection, and analytical techniques used. Case studies, pedagogical articles, book reviews, debates, and opinion papers will be considered but will not make the bulk of the journal. Articles with a high technical and low managerial content are not encouraged but may be accepted if highly relevant or innovative. Articles may not include more than 8,000 words. 